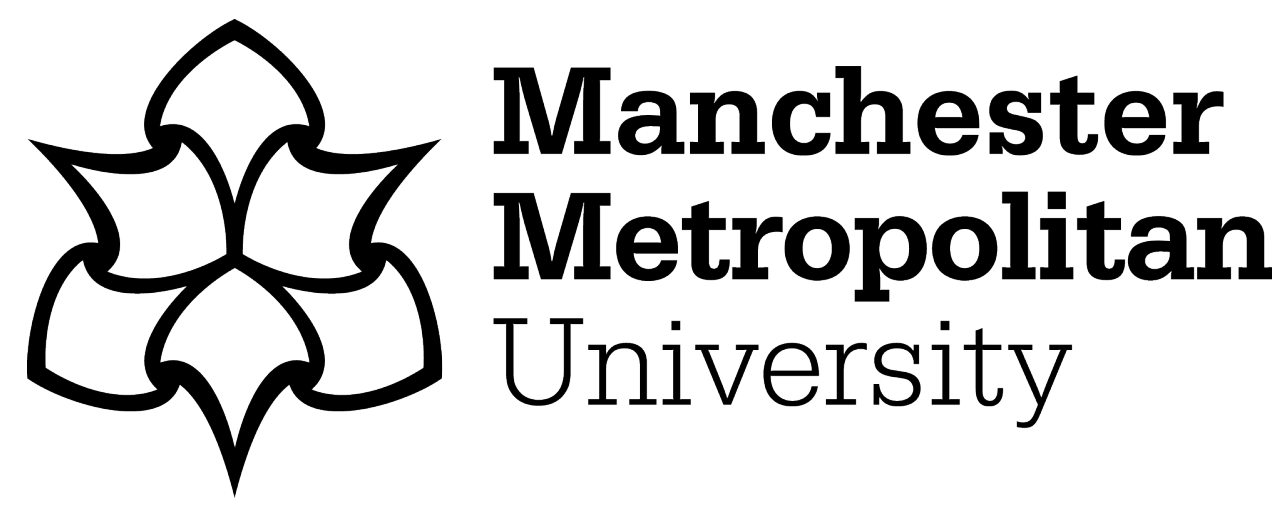

Daniels, Katherine AJ, King, Enda, Richter, Chris, Falvey, Éanna and Franklyn-Miller, Andy (2021) Changes in the kinetics and kinematics of a reactive cut manoeuvre after successful athletic groin pain rehabilitation. Scandinavian Journal of Medicine and Science in Sports, 31 (4). pp. 839-847. ISSN 0905-7188

Downloaded from: https://e-space.mmu.ac.uk/626802/

Version: Accepted Version

Publisher: Wiley

DOI: https://doi.org/10.1111/sms.13860

Please cite the published version 


\title{
Changes in the kinetics and kinematics of a reactive cut manoeuvre after successful athletic groin pain rehabilitation
}

Katherine A J Daniels ${ }^{1,2,3}$, Enda King ${ }^{1,4}$, Chris Richter ${ }^{1,4}$, Éanna Falvey ${ }^{1,5}$ and Andy FranklynMiller ${ }^{1,6}$

${ }^{1}$ Sports Medicine Research Department, Sports Surgery Clinic, Dublin, Ireland

${ }^{2}$ Queen's School of Engineering, University of Bristol, Bristol, UK

${ }^{3}$ Department of Sport and Exercise Sciences, Manchester Metropolitan University, Manchester, UK

${ }^{4}$ Department of Life Sciences, University of Roehampton, London, UK

${ }^{5}$ Department of Medicine, University College Cork, Cork, Ireland

${ }^{6}$ Centre for Health, Exercise and Sports Medicine, University of Melbourne, Melbourne, Australia

\section{Corresponding author:}

Katherine A J Daniels

Department of Sport and Exercise Sciences

Manchester Metropolitan University

Manchester

$U K$

Email:k.daniels@mmu.ac.uk

\begin{abstract}
Athletic groin pain (AGP) is a chronic, painful condition which is prevalent in players of field sports that require rapid changes of direction. Following successful rehabilitation, systematic changes have been observed in the kinetics and kinematics of pre-planned change of direction manoeuvres, providing insight into potential foci for rehabilitation monitoring and for the assessment of interventions. However, changing direction in field sports is often reactive rather than pre-planned, and it is not known whether such post-rehabilitation changes are seen in reactive manoeuvres. We analysed the stance phase kinetics and kinematics of a $90^{\circ}$ reactive cutting manoeuvre in 35 AGP patients before and after a successful exercise intervention programme. Following the intervention, transverse plane rotation of the pelvis towards the intended direction of travel increased, and the body centre of mass was
\end{abstract}


positioned more anteriorly relative to the centre of pressure. Ankle dorsiflexion also increased, and participants demonstrated greater ankle plantar flexor internal moment and power during the second half of stance. These findings provide insight into mechanical variables of potential importance in AGP, as identified during a manoeuvre based on a common sporting task.

\section{Key words}

Biomechanics, intervention, change of direction, injury, performance

\section{Introduction}

Athletic groin pain (AGP) is a prevalent pain presentation in field sports involving activities such as sprinting, kicking, acceleration and rapid changes of direction ${ }^{1-8}$. Affected athletes present with painful structures around the groin and anterior pelvic area, which are commonly aggravated by dynamic loading of bony and musculotendinous tissue in the region ${ }^{9}$. Multiple risk factors have been reported, including sport, playing position, training history and hip adductor strength ${ }^{10,11}$, but the aetiology and optimal treatment of the condition remain poorly understood because AGP often follows a protracted sub-acute presentation, during which athletes continue to train and play ${ }^{1,12}$, and has a high recurrence rate ${ }^{13}$.

Given the preceding sub-acute profile and recurrence rates, recent studies have examined multi-planar whole-body movement to investigate potential injury mechanisms and monitor changes after a rehabilitation intervention to better understand the biomechanics associated with the presence of and recovery from AGP ${ }^{14-19}$. The main focus of the research in this area to date has been on change of direction manoeuvres, as these are ubiquitous in field sports and most-commonly noted as provocative in AGP populations ${ }^{4,20,21}$, but jumping and landing 
tasks have also been investigated ${ }^{17-19}$. Many of the reported prospective risk factors for AGP are associated with impaired neuromuscular function and joint excursion at the hip, such as weakness in the adductor musculature and decreased range of motion. These factors might be expected to translate into altered kinematics and kinetics during athletic tasks due to the relationship between impaired isolated joint-level neuromuscular function and modified whole-body segmental dynamics that has been demonstrated for a variety of impairments ${ }^{22,23}$. As the forces applied to body tissues are dependent on the kinematic relationships between segments, any deficits in strength or control would be expected to manifest themselves across multiple joints and structures ${ }^{24,25}$. This premise is supported by the findings of studies investigating change of direction manoeuvres in AGP: differences in kinetic and kinematic variables in all three planes were identified across the hip, knee, trunk and ankle following rehabilitation ${ }^{14,17}$ and when comparing those with and without a history of AGP ${ }^{16,17}$.

Compensatory offloading of weak or painful structures can involve redistribution of work to other joints ${ }^{26,27}$ and to the contralateral limb ${ }^{28}$. In particular, frontal plane control of the torso has been implicated in a wide variety of lower limb injuries in multidirectional sports, due to the large influence of this relatively high-mass segment on joint torques ${ }^{29-31}$. More recently, ankle plantar flexor moments and powers in jumping and cutting tasks have been found to increase concomitantly with a reduction in AGP symptoms, and to be lower in those presenting with AGP than in healthy controls, with corresponding deficits in ankle sagittal plane joint quasi-stiffness ${ }^{14,17,19}$. The ankle joint has been shown to be the greatest contributor to mechanical energy absorption in single-leg landings, modulating the loads experienced at more-proximal joints ${ }^{32,33}$. Reductions in ankle stiffness may indicate a compensatory mechanism to reduce peak forces, or may represent a lack of capacity to 
generate high forces quickly ${ }^{17}$. Regardless, lower angle plantar flexor moments and powers are associated with slower cut completion times in uninjured athletes ${ }^{34}$, showing evident correlation with reduced performance. A recent exploratory study in a large cohort of AGP patients performing a pre-planned $110^{\circ}$ cutting manoeuvre before and after successful rehabilitation identified changes in twenty-eight biomechanical variables including reduced lateral trunk flexion, increased pelvis rotation in the direction of travel, increased hip flexion, reduced knee flexion and increased ankle plantar flexor moment ${ }^{14}$. These results not only emphasise the potential role of cutting movement strategy in AGP but also highlight the potential value of this approach in identifying foci for rehabilitation and evaluating interventions.

Cutting manoeuvres in field sports are often reactive, as athletes respond to the movement of other players and of the ball. Reactive cutting tasks are associated with greater lower-limb joint moments than pre-planned tasks ${ }^{35-37}$ and are likely to reflect the movement strategies implemented during play more closely ${ }^{38}$. Analysis of reactive cutting biomechanics in AGP patients may thus highlight variables that are more representative of the movement patterns exhibited in the real-world athletic activities that originally induced symptoms and are the focus for return to sport. Changes associated with successful rehabilitation hence provide potential key targets for AGP intervention and metrics for rehabilitation progress monitoring.

The aim of this study was to investigate changes in kinematic, kinetic and performance variables in AGP patients performing a reactive cutting manoeuvre following a successful rehabilitation intervention focused on intersegmental control, linear running and change of direction mechanics. We hypothesised based on previous findings in pre-planned cutting tasks that we would find (a) improved trunk control as characterised by increased body 
rotation in the direction of travel and reduced frontal plane lateral flexion; (b) increased sagittal plane ankle dorsiflexion, moments and power; and (c) faster manoeuvre completion times after the intervention.

\section{Methods}

\section{Participants}

Thirty-five male recreational multi-directional field sport players with an anatomical diagnosis falling under $\mathrm{AGP}^{39}$ (mean \pm SD: age $24.9 \pm 5.8$ years; height $180.6 \pm 6.3 \mathrm{~cm}$; mass $78.7 \pm 8.6 \mathrm{~kg}$ ) who had presented at Sports Surgery Clinic, Dublin, Ireland participated in this study. This cohort comprised all those who met the eligibility criteria and had completed a laboratory testing session prior to a rehabilitation intervention, successfully completed the rehabilitation intervention programme (i.e. return to play criteria met as per ${ }^{14}$ ) and lastly completed a final laboratory testing session between May 2015 and June 2016. Sites of pain were rectus aponeurosis (55\%), adductor (26\%), iliopsoas (26\%) and hip (23\%). The primary sports played by the participants were Gaelic football (49\%), soccer (23\%), hurling (14\%) and rugby (14\%). We planned to report only post-intervention biomechanical changes with a standardised effect size (Cohen's d) of 0.5 (medium) ${ }^{40}$ or above, given the exploratory nature of the study, to minimise the over-interpretation of small differences. We powered the study to detect point effects of this magnitude: a sample size of 34 is required to achieve $80 \%$ statistical power with an alpha level of 0.05 for a Cohen's $d$ of 0.5 . As we lacked an a priori framework to specify the function of the expected effect we were not able to calculate a sample size based on power for comparing curves, but the required sample size would be expected to have been overestimated rather than underestimated. Median selfreported duration of groin pain at the time of the first testing session was 28 weeks (IQR 16- 
52 weeks). Informed written consent was obtained from all participants and ethical approval for the study was obtained from the Sports Surgery Clinic Hospital Ethics committee (25EF011).

\section{Data collection}

Participants completed a $90^{\circ}$ maximum-effort reactive cutting task before (PRE) and after (POST) an exercise rehabilitation intervention. The task involved running towards a stationary mannequin, responding to a visual signal to indicate the direction of the manoeuvre (left or right), cutting in the indicated direction and finally passing through a second gate (Gate 2 or 3 ) located $2 \mathrm{~m}$ from the mannequin in the new direction of travel (Figure 1). The visual signal was given $4 \mathrm{~m}$ before reaching the mannequin (as the participant passed through Gate 1) and the direction was selected randomly for each trial, continuing until three valid trials had been collected in each direction. Total time for the manoeuvre from onset of visual signal to passing through the final gate was recorded using photoelectric cells (SMARTSPEED timing gates system, Fusion Sport, QLD, Australia). All participants wore their own athletic running footwear and completed a standardised warm-up routine comprised of jogging, squats and jumps prior to testing. The reactive change of direction task was preceded by a battery of tests used for clinical assessment: double and single-leg drop landings, hurdle hops and a $110^{\circ}$ pre-planned cutting manoeuvre. Participants then completed two sub-maximal and one maximal-effort practice trial before data collection commenced. Retroreflective markers of $14 \mathrm{~mm}$ diameter were positioned on the lower limbs, pelvis and trunk of the participant in accordance with a modified Plug-In Gait marker set ${ }^{34}$. A tencamera optical motion capture system (Bonita B10, Vicon Motion Systems Ltd, Oxon, UK) was synchronised with two force platforms (BP400600, AMTI, MA, USA) and used to record ground reaction forces (GRFs), including the location of the centre of pressure (COP), 
and the positions of the markers at 1000 samples/s and 200 samples/s respectively during each trial.

The rehabilitation programme consisted of three levels: Level 1 focused on intersegmental control and strength, Level 2 on linear running mechanics and load tolerance and Level 3 on sprinting and multidirectional mechanics. Competency was achieved at each level before progression to the next, and participants were able to undertake multi-directional drills painfree at maximal intensity before being cleared to return to play and returning for POST testing. An extensive description of the rehabilitation programme, progression criteria and return to play criteria is given in ${ }^{14}$. Briefly, the criteria for progression and return to play clearance were negative crossover sign (progression from Level 1 to Level 2); symmetrical hip internal rotation at $90^{\circ}$, pain-free squeeze at $45^{\circ}$ and symptom-free completion of a progressive linear running programme (progression from Level 2 to Level 3); and symptomfree completion of a sprints and multidirectional drills programme with all tasks completed at maximum intensity (return to play clearance and post-intervention laboratory testing). Median time between PRE and POST testing sessions was 10.9 weeks (IQR 8.6 - 12.8 weeks). Participants completed the Copenhagen Hip And Groin Outcome Score (HAGOS) questionnaire (Thorborg, Hölmich, Christensen, Petersen, \& Roos, 2011) at the time of each testing session.

\section{Data processing and analysis}

Only trials in which the participants used the symptomatic side as the stance leg for the cut manoeuvre were analysed. GRF and marker position data were filtered using a fourth-order zero-lag Butterworth filter with a corner frequency of $15 \mathrm{~Hz}{ }^{42}$. Motion capture data were processed using the Vicon Plug-In Gait model to calculate joint and segment mechanics 
(Nexus 2.3, Vicon Motion System Ltd, UK) by applying standard inverse dynamics techniques ${ }^{43}$. Moments are reported as internal net joint moments. Joint power was calculated in each plane as the product of joint moment and angular velocity. Kinetic data were normalised to body mass. Stance phase contact time was identified by the start and end of the GRF signal $(>10 \mathrm{~N})$ and kinematic and kinetic waveforms were then time-normalised to 101 data points using a dynamic time warping process ${ }^{44}$ to align the end of the braking phase across all curves.

Biomechanical variables analysed were: angle, moment and power at the hip, knee and ankle joints in the sagittal, frontal and transverse planes; pelvis angles relative to the laboratory (global) axis system; thorax angle relative to pelvis angle in the sagittal, frontal and transverse planes; GRF in the laboratory x direction (Figure 1); GRF in the laboratory y direction; horizontal distance from the body centre of mass $(\mathrm{COM})$ to the centre of pressure $(\mathrm{COP})$ in the sagittal plane and the frontal plane. Performance variables were: total time to complete the manoeuvre (Gate 1 to final gate), contact time (duration of the cut step stance phase), horizontal (x-y plane) velocity of the $\mathrm{COM}\left(\mathrm{V}_{\mathrm{COMH}}\right)$ at stance initial contact and $\mathrm{V}_{\mathrm{COMH}}$ at stance toe-off.

Paired Student's $t$ tests were used to test the null hypotheses that performance variables and HAGOS subscale scores did not differ between PRE and POST. Statistical parametric mapping (SPM; 1D paired t test) was used to identify differences between PRE and POST biomechanical variables for the symptomatic side from 0 to $100 \%$ stance phase. Significance was accepted at $\alpha=0.05$. Cohen's $d$ effect size ${ }^{40}$ was calculated in a point-by-point manner for differences (0.2-0.5 small; 0.5-0.8 moderate; >0.8 strong) using the time-normalised signal and only significant differences with Cohen's d $>0.5$ were reported. All statistical analyses were performed using MATLAB (R2015a, MathWorks, USA). 


\section{Results}

Significant changes with moderate effect sizes were identified in kinematic and kinetic biomechanical variables during stance phase (Table 1;
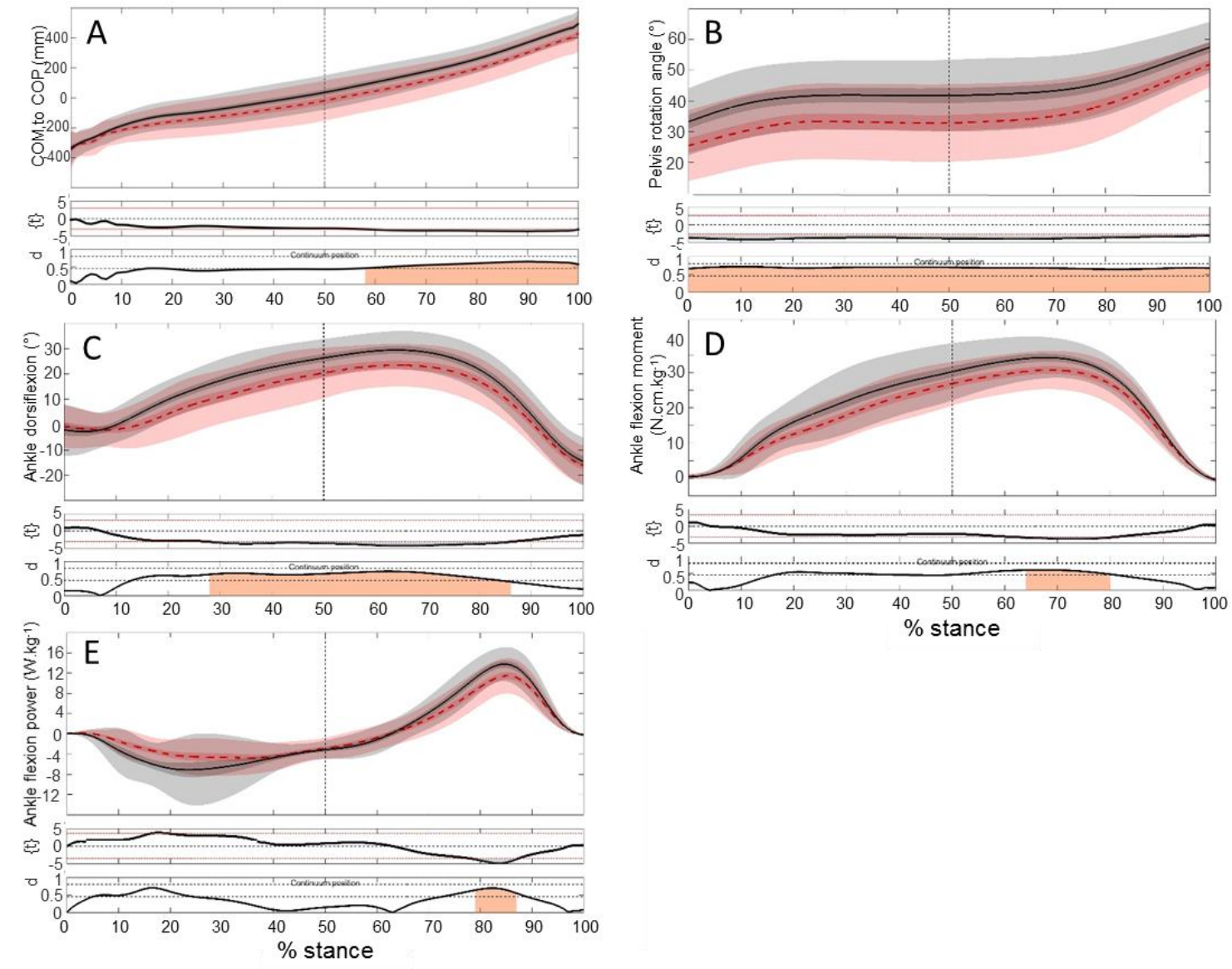

Figure 2). POST-intervention, the COM was positioned more anteriorly relative to the COP (58-100\% stance phase). Rotation of the pelvis in the transverse plane towards the direction of intended travel increased POST-intervention across stance (0-100\%). Ankle dorsiflexion 
also increased (27-100\% stance phase) and participants demonstrated greater ankle plantar flexor internal moment and power during the second half of stance (
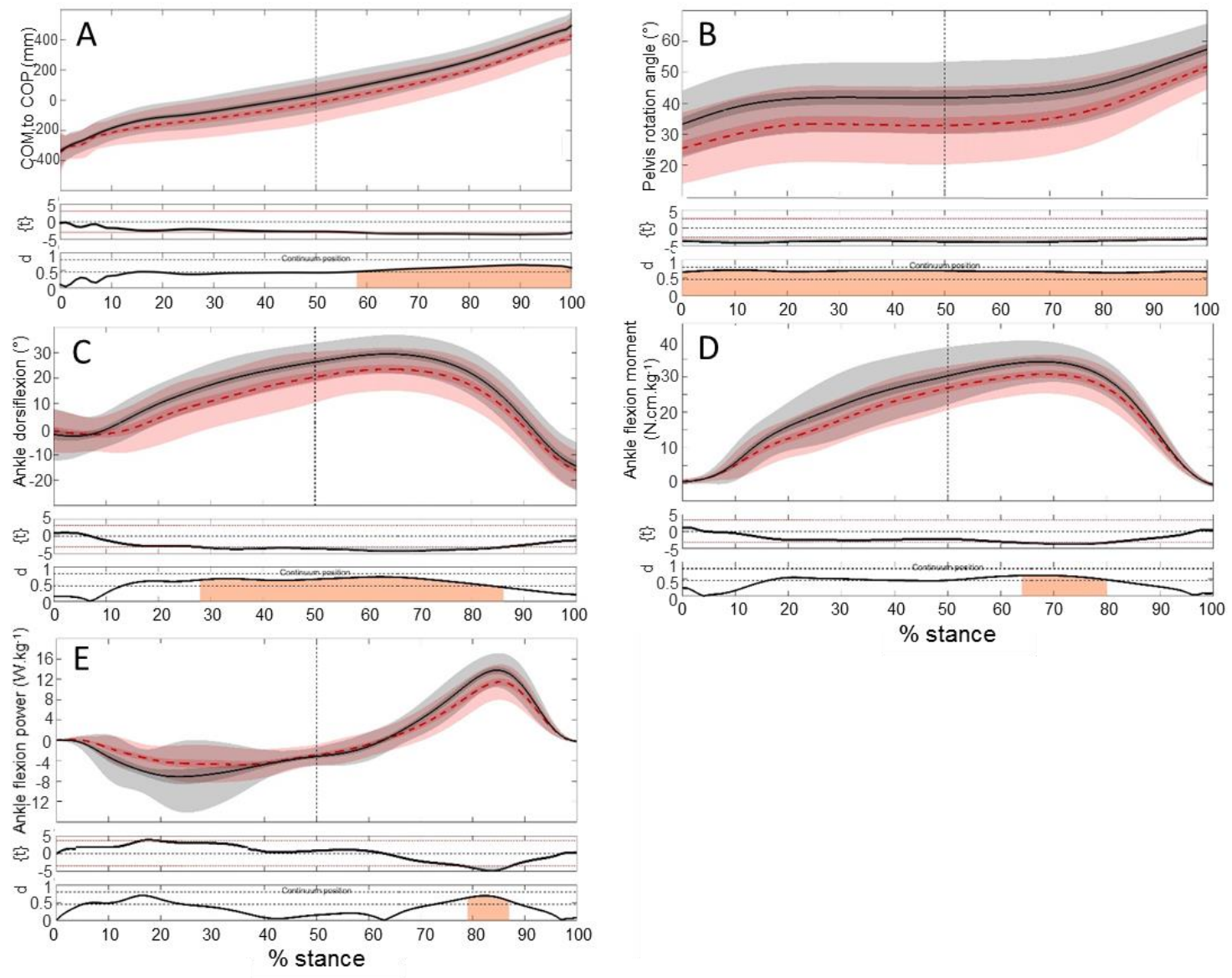

Figure 2). We note that we did not find any additional biomechanical variables with significant PRE-POST changes and effect sizes below 0.5: the variables reported in Table 1 are the only ones for which significant differences of any effect size were identified. The results and graphs from PRE-POST comparisons for all kinematic and kinetic continuous biomechanical variables where no significant differences were found are provided as a Supplementary file. No performance variables were found to alter significantly from PRE to POST (Table 2) and effect sizes ranged from 0 to 0.27 (trivial to small).

All subscales of HAGOS (Pain, Symptoms, Function in Activities of Daily Living, Function 
in Sport and Recreation, Participation in Physical Activities and Quality of Life) improved significantly PRE to POST ( $<<0.001$, Cohen's d 0.91-1.89; Table 3).

\section{Discussion}

We found significant changes in kinematics and kinetics, but not in performance variables, following the rehabilitation intervention. The identified post-rehabilitation increase in rotation of the pelvis towards the intended direction of travel, more-anterior position of the COM relative to the COP and increase in ankle dorsiflexion, moment and power were all also seen in a pre-planned $110^{\circ}$ cut in a different experimental cohort ${ }^{14}$, indicating commonality of post-rehabilitation changes across these manoeuvres despite the difference in change of direction angle and anticipation status.

It is notable that the majority of identified changes in biomechanical variables, including all changes in kinetic variables, were at the ankle joint. The ankle both modulates the transmission of GRFs to proximal structures and is mechanically influenced by the relatively high-mass segments of the upper body and proximal limb ${ }^{45,46}$. Greater ankle plantar flexor power and moment, as observed post-rehabilitation, have previously been associated with a reduction in time to complete pre-planned cutting manoeuvres ${ }^{34,47}$ and demonstrated an increased ability to generate force quickly around the ankle in the sagittal plane. The COM was in a more anterior position relative to the COP post-rehabilitation, with a corresponding increase in ankle dorsiflexion evident for the majority of stance. Greater dorsiflexion has been reported to increase the ability to generate high forces in the ankle plantar flexor musculature ${ }^{48}$. Similar exercises to those incorporated into the rehabilitation intervention (described in detail in King et al. $2018^{14}$ ) have been found to increase isokinetic plantar flexor power and peak torque in various experimental populations ${ }^{49-51}$ so the intervention 
might be expected to have developed these qualities. Recent work using a lateral jumping task to understand the effects of AGP rehabilitation in a similar cohort also identified greater ankle sagittal plane moments and powers after successful rehabilitation ${ }^{17}$, suggesting that these changes are not task-specific but instead more-generally representative of either increased neuromuscular capacity or of decreased protective need to limit peak forces. As strength and neuromuscular control at the ankle joint influence the dynamics of the proximal segments ${ }^{52}$, this study suggests that ankle biomechanics may be a potentially-important focus for improving outcomes in AGP rehabilitation.

Previous investigations examining factors associated with sport-related pain in the hip and groin region have typically focused on variables appertaining to the pelvis, hip and torso 10,11,53. We did not identify frontal plane changes indicative of reduced pelvis and thorax lateral tilt or lean after rehabilitation, despite both having previously been found with medium to large effect sizes (0.62 and 0.79$)$ in a similar pre-planned cutting manoeuvre ${ }^{14}$, and also did not identify any changes at the hip joint. The lack of hip and torso-related biomechanical changes identified here, in contrast to previous findings for pre-planned tasks in similar populations ${ }^{14,17}$, could be explained by between-study differences in the change of direction angle investigated or may be demonstrative of the differing demands present in the reactive cut $^{35,54}$. We also did not identify significant differences in performance variables (although the $\mathrm{p}$ value for total time was only just outside the threshold for significance), and effect sizes were trivial to small, suggesting that the time and speed measures utilised may not be useful markers of AGP rehabilitation status in this manoeuvre and also that the biomechanical changes observed are unlikely to be simply correlates of altered speed ${ }^{34,47,55}$.

Our analysis was not constrained to variables for which differences had previously been 
identified in pre-planned cutting, due to limited previous studies relating AGP to reactive cutting biomechanics and the poor correlations between pre-planned and reactive cutting ${ }^{56,57}$, so we chose to report only post-intervention biomechanical changes with a Cohen's d standardised effect size of 0.5 (medium) or above to reduce over-interpretation of small differences. However, it is not necessarily the case that the largest standardised effect sizes correspond to the most clinically important differences, and thus an important focus for future studies is to build on this exploratory work and develop greater mechanistic insight into the relationship between biomechanics and AGP. Although our results demonstrate that biomechanical changes following improvement in AGP symptoms can be observed in this reactive manoeuvre, the study design does not enable discrimination between AGP as a cause and as a consequence of the pre-intervention biomechanics. It also does not enable the improvement in symptoms and PRE-POST movement strategy changes to be conclusively ascribed to the rehabilitation intervention, although the 28-week median average symptom duration (IQR 16-52 weeks) prior to the PRE testing session suggests that the intervention is likely to have had an effect on outcomes. The term AGP is used inclusive of all areas of palpatory tenderness described in the Doha agreement on terminology of hip and groin pain ${ }^{58}$. However, the majority of the participants' main site of palpatory pain on resisted sit up was an area superior to the adductor tubercle but below the pubic tubercle, which we refer to as rectus aponeurosis. This area of palpatory tenderness is not covered in the Doha agreement and the palpatory anatomy hence requires further explanation and differentiation.

Although the manoeuvre involved a reactive component in terms of the direction of the turn, the participant was aware that a change of direction would be required so the task was not fully unanticipated, limiting direct comparability to many sporting situations. Reactive cutting mechanics are known to be affected by the nature and timing of the stimuli used to 
indicate the direction of the turn ${ }^{59,60}$, so altering the time available to prepare for the manoeuvre or the type of stimulus presented may have reduced, accentuated or altered the changes we identified. The presence of separate sub-groups with distinct movement patterns in the AGP population ${ }^{15}$ may have masked additional changes in outcome variables and future studies should investigate whether this is the case.

\section{Conclusion}

Systematic changes in the kinetics and kinematics of a $90^{\circ}$ reactive cutting manoeuvre were found in patients previously diagnosed with AGP following a successful conservative exercise-based rehabilitation intervention. All identified changes were also previously observed in a pre-planned $110^{\circ}$ cut in a different experimental cohort ${ }^{14}$, suggesting that these post-rehabilitation modifications to movement are robust to differences in anticipation status. The localisation of kinetic changes to the ankle joint is notable, and suggests that the role of altered distal limb mechanics as a cause or consequence of AGP may warrant further investigation.

\section{Perspective}

Whilst previous studies have indicated that kinetic and kinematic changes in various pre-planned movement tasks can be observed following successful rehabilitation of athletic groin pain, this is the first time such changes have been demonstrated in an unplanned reactive sport-specific manoeuvre. The differences in pelvis rotation and COM positioning relative to foot placement are suggestive of improved upper body control, whilst the increased ankle sagittal plane moment and power in the second half of stance phase provides insight into the potential role of distal limb function in athletic groin pain. Our findings highlight potential key targets for AGP intervention and 
metrics for both monitoring rehabilitation progress in athletes and evaluating interventions.

\section{Conflict of Interest}

The authors have no conflict of interest to declare.

\section{References}

1. Waldén $M$, Hägglund $M$, Ekstrand J. The epidemiology of groin injury in senior football: a systematic review of prospective studies. Br J Sports Med. 2015;49(12):792-797. doi:10.1136/bjsports-2015-094705

2. O'Connor D. Groin injuries in professional rugby league players: a prospective study. J Sports Sci. 2004;22(7):629-636. doi:10.1080/02640410310001655804

3. Brooks JHM. Epidemiology of injuries in English professional rugby union: part 2 training Injuries. Br J Sports Med. 2005;39(10):767-775. doi:10.1136/bjsm.2005.018408

4. Emery CA, Meeuwisse W, Powell J. Groin and abdominal strain injuries in the National Hockey League. Clin J Sport Med. 1999;9:151-156.

5. Brooks JHM. Epidemiology of injuries in English professional rugby union: part 1 match injuries. Br J Sports Med. 2005;39(10):757-766. doi:10.1136/bjsm.2005.018135

6. Orchard J, Seward H. Epidemiology of injuries in the Australian Football League, seasons 1997-2000. Sport Med. 2002;36:39-45.

7. Wilson F, Caffrey S, King E, Casey K, Gissane C. A 6-month prospective study of injury in Gaelic football. Br J Sports Med. 2007;41(5):317-321. doi:10.1136/bjsm.2006.033167

8. Murphy JC, O'Malley E, Gissane C, Blake C. Incidence of Injury in Gaelic Football: A 4-Year Prospective Study. Am J Sports Med. 2012;40(9):2113-2120. doi:10.1177/0363546512455315

9. Hölmich P, Thorborg K, Dehlendorff C, Krogsgaard K, Gluud C. Incidence and clinical presentation of groin injuries in sub-elite male soccer. Br J Sports Med. 2014;48(16):12451250. doi:10.1136/bjsports-2013-092627

10. Whittaker JL, Small C, Maffey L, Emery CA. Risk factors for groin injury in sport: an updated systematic review. Br J Sports Med. 2015;49:803-809. doi:10.1136/bjsports-2014-094287

11. Ryan J, Deburca N, Creesh KM. Risk factors for groin/hip injuries in field-based sports: a systematic review. Br J Sports Med. 2014;(0):1-8. doi:10.1136/bjsports-2013-092263

12. Esteve $\mathrm{E}, \mathrm{Clausen} \mathrm{MB}$, Rathleff MS, et al. Prevalence and severity of groin problems in Spanish football: A prospective study beyond the time-loss approach. Scand J Med Sci Sport.

2019;30(5):914-921. doi:10.1111/sms.13615 
13. Arnason A, Sigurdsson SB, Gudmundsson A, Holme I, Engebretsen L, Bahr R. Risk Factors for Injuries in Football. Am J Sports Med. 2004;32(SUPPL. 1):5-16.

doi:10.1177/0363546503258912

14. King E, Franklyn-Miller A, Richter C, et al. Clinical and biomechanical outcomes of rehabilitation targeting intersegmental control in athletic groin pain: prospective cohort of 205 patients. Br J Sports Med. 2018;52:1054-1062. doi:10.1136/bjsports-2016-097089

15. Franklyn-Miller A, Richter C, King E, et al. Athletic groin pain (part 2): A prospective cohort study on the biomechanical evaluation of change of direction identifies three clusters of movement patterns. Br J Sports Med. 2017;51(5):460-468. doi:10.1136/bjsports-2016-096050

16. Edwards S, Brooke HC, Cook JL. Distinct cut task strategy in Australian football players with a history of groin pain. Phys Ther Sport. 2017;23:58-66. doi:10.1016/j.ptsp.2016.07.005

17. Gore S, Franklyn-Miller A, Richter C, King E, Falvey EC, Moran K. The effects of rehabilitation on the biomechanics of patients with athletic groin pain. J Biomech. 2020;23(99):109474. doi:10.1016/J.JBIOMECH.2019.109474

18. Janse van Rensburg L, Dare $M$, Louw $Q$, et al. Pelvic and hip kinematics during single-leg droplanding are altered in sports participants with long-standing groin pain: A cross-sectional study. Phys Ther Sport. 2017;26:20-26. doi:10.1016/j.ptsp.2017.05.003

19. Gore SJ, Franklyn-Miller A, Richter C, Falvey EC, King E, Moran K. Is stiffness related to athletic groin pain? Scand J Med Sci Sport. 2018;28(6):1681-1690. doi:10.1111/sms.13069

20. Kloskowska P, Morrissey D, Small C, Malliaras P, Barton C. Movement Patterns and Muscular Function Before and After Onset of Sports-Related Groin Pain: A Systematic Review with Meta-analysis. Sport Med. 2016;46(12):1847-1867. doi:10.1007/s40279-016-0523-z

21. Estwanik JJ, Sloane B, Rosenberg MA. Groin Strain and Other Possible Causes of Groin Pain. Phys Sportsmed. 1990;18(2):54-65. doi:10.1080/00913847.1990.11709972

22. Weinhandl JT, Earl-Boehm JE, Ebersole KT, Huddleston WE, Armstrong BSR, O'Connor KM. Reduced hamstring strength increases anterior cruciate ligament loading during anticipated sidestep cutting. Clin Biomech. 2014;29(7):752-759. doi:10.1016/j.clinbiomech.2014.05.013

23. Oberländer KD, Brüggemann GP, Höher J, Karamanidis K. Altered landing mechanics in ACLreconstructed patients. Med Sci Sports Exerc. 2013;45(3):506-513. doi:10.1249/MSS.0b013e3182752ae3

24. Havens KL, Sigward SM. Joint and segmental mechanics differ among running and cutting maneuvers in skilled athletes. Gait Posture. 2015;41(1):33-38.

doi:10.1016/j.gaitpost.2014.07.022

25. Winter D. Human balance and posture control during standing and walking. Gait Posture. 1995;3(4):193-214. doi:10.1016/0966-6362(96)82849-9

26. Orishimo KF, Kremenic IJ, Mullaney MJ, McHugh MP, Nicholas SJ. Adaptations in single-leg hop biomechanics following anterior cruciate ligament reconstruction. Knee Surgery, Sport Traumatol Arthrosc. 2010;18(11):1587-1593. doi:10.1007/s00167-010-1185-2

27. Decker MJ, Torry MR, Noonan TJ, Riviere A, Sterett WI. Landing adaptations after ACL reconstruction. Med Sci Sports Exerc. 2002;34(9):1408-1413. doi:10.1097/00005768200209000-00002 
28. Miles JJ, King E, Falvey ÉC, Daniels KAJ. Patellar and hamstring autografts are associated with different jump task loading asymmetries after ACL reconstruction. Scand J Med Sci Sports. 2019;0:1-11. doi:10.1111/sms.13441

29. Dempsey AR, Lloyd DG, Elliott BC, Steele JR, Munro BJ, Russo KA. The effect of technique change on knee loads during sidestep cutting. Med Sci Sports Exerc. 2007;39(10):1765-1773. doi:10.1249/mss.0b013e31812f56d1

30. Frank B, Bell DR, Norcross MF, Blackburn JT, Goerger BM, Padua DA. Trunk and hip biomechanics influence anterior cruciate loading mechanisms in physically active participants. Am J Sports Med. 2013;41(11):2676-2683. doi:10.1177/0363546513496625

31. Jones PA, Herrington LC, Graham-Smith P. Technique determinants of knee joint loads during cutting in female soccer players. Hum Mov Sci. 2015;42:203-211.

doi:10.1016/j.humov.2015.05.004

32. Yeow $\mathrm{CH}$, Lee PVS, Goh JCH. An investigation of lower extremity energy dissipation strategies during single-leg and double-leg landing based on sagittal and frontal plane biomechanics. Hum Mov Sci. 2011;30(3):624-635. doi:10.1016/j.humov.2010.11.010

33. Chinnasee C, Weir G, Sasimontonkul S, Alderson J, Donnelly C. A biomechanical comparison of single-leg landing and u sidestepping. Int J Sports Med. 2018;39(8):636-645.

34. Marshall BM, Franklyn-Miller AD, King E, et al. Biomechanical factors associated with time to complete a change of direction cutting maneuver. J Strength Cond Res. 2014;28(10):28452851. doi:10.1519/JSC.0000000000000463

35. Brown SR, Brughelli M, Hume PA. Knee Mechanics During Planned and Unplanned Sidestepping: A Systematic Review and Meta-Analysis. Sport Med. 2014;44(11):1573-1588. doi:10.1007/s40279-014-0225-3

36. Almonroeder TG, Garcia E, Kurt M. The Effects of Anticipation on the Mechanics of the Knee During Single-Leg Cutting Tasks: A Systematic Review. Int J Sports Phys Ther. 2015;10(7):918928.

37. Meinerz CM, Malloy P, Geiser CF, Kipp K. Anticipatory Effects on Lower Extremity Neuromechanics During a Cutting Task. J AthI Train. 2015;50(9):905-913. doi:10.4085/10626050-50.8.02

38. O'Connor K, Monteiro SK, Hoelker IA. Comparison of Selected Lateral Cutting Activities Used to Assess ACL Injury Risk. J Appl Biomech. 2009;25:9-21.

39. Falvey ÉC, King E, Kinsella S, Franklyn-Miller A. Athletic groin pain (part 1): a prospective anatomical diagnosis of 382 patients-clinical findings, MRI findings and patient-reported outcome measures at baseline. Br J Sports Med. 2016;50(7):423-430. doi:10.1136/bjsports2015-094912

40. Cohen J. A Power Primer. Psychol Bull. 1992;112(1):155-159. doi:10.1037/00332909.112.1.155

41. Thorborg K, Hölmich P, Christensen R, Petersen J, Roos EM. The Copenhagen Hip and Groin Outcome Score (HAGOS): development and validation according to the COSMIN checklist. $\mathrm{Br} J$ Sports Med. 2011;45(6):478-491. doi:10.1136/bjsm.2010.080937

42. Kristianslund E, Krosshaug T, Van den Bogert AJ. Effect of low pass filtering on joint moments 
from inverse dynamics: Implications for injury prevention. J Biomech. 2012;45(4):666-671. doi:10.1016/j.jbiomech.2011.12.011

43. Winter D. Biomechanics and Motor Control of Human Movement. 3rd editio. Hoboken: John Wiley and Sons, Inc.; 2005.

44. Ramsey J. Functional Data Analysis. John Wiley and Sons, Inc.; 2006.

45. Saha D, Gard S, Fatone S. The effect of trunk flexion on able-bodied gait. Gait Posture. 2008;27(4):653-660.

46. Kluger D, Major MJ, Fatone S, Gard SA. The effect of trunk flexion on lower-limb kinetics of able-bodied gait. Hum Mov Sci. 2014;33(1):395-403. doi:10.1016/j.humov.2013.12.006

47. Havens KL, Sigward SM. Cutting mechanics: Relation to performance and anterior cruciate ligament injury risk. Med Sci Sports Exerc. 2015;47(4):818-824.

doi:10.1249/MSS.0000000000000470

48. Herzog W, Read LJ, Keurs HEDJ. Experimental determination of force-length relations of intact human gastrocnemius muscles. Clin Biomech. 1991;6:230-238.

49. Beijersbergen CMI, Granacher U, Gabler M, Devita P, Hortobagyi T. Kinematic Mechanisms of How Power Training Improves Healthy Old Adults' Gait Velocity. Med Sci Sports Exerc. 2016;49(1):150-157. doi:10.1249/MSS.0000000000001082

50. Jeon K-K, Kim T-Y, Lee S-H. The effects of a strategic strength resistance exercise program on the isokinetic muscular function of the ankle. J Phys Ther Sci. 2015;27(10):3295-3297.

51. Marín-Cascales E, Alcaraz P, Rubio-Arias J. Effects of 24 weeks of whole body vibration vs. multi-component training on muscle strength and body composition in postmenopausal women: a randomized controlled trial. Rejuvination Res. 2017;20(3):193-201. doi:10.1089/rej.2016.1877

52. Jeon K-K, Chun S-Y, Seo B. Effects of muscle strength asymmetry between left and right on isokinetic strength of the knee and ankle joints depending on athletic performance level. $J$ Phys Ther Sci. 2016;28:1289-1293.

53. Sedaghati $P$, Alizadeh $M$, Shirzad E, Ardjmand A. Review of sport-induced groin injuries. Trauma Mon. 2013;18(3):107-112. doi:10.5812/traumamon.12666

54. Kim JH, Lee K, Kong SJ, An KO. Effect of Anticipation on Lower Extremity Biomechanics During Side- and Cross- Cutting Maneuvers in Young Soccer Players. Am J Sports Med. 2014;42(8):1985-1992. doi:10.1177/0363546514531578

55. Smith N, Dyson R, Hale T, Janaway L. Contributions of the inside and outside leg to maintenance of curvilinear motion on a natural turf surface. 2006;24:453-458. doi:10.1016/j.gaitpost.2005.11.007

56. Houck JR, Duncan A, Haven KE De. Comparison of frontal plane trunk kinematics and hip and knee moments during anticipated and unanticipated walking and side step cutting tasks. Gait Posture. 2006;24(3):314-322. doi:10.1016/j.gaitpost.2005.10.005

57. Gabbett TJ, Kelly JN, Sheppard JM. Speed, Change of Direction Speed, and Reactive Agility of Rugby League Players. J Strength Cond Res. 2008;22(1):174-181. doi:10.1519/JSC.0b013e31815ef700 
58. Weir A, Brukner P, Delahunt E, et al. Doha agreement meeting on terminology and definitions in groin pain in athletes. Br J Sports Med. 2015;49(12):768-774. doi:10.1136/bjsports-2015094869

59. Lee MJC, Lloyd DG, Lay BS, Bourke PD, Alderson JA. Different visual stimuli affect body reorientation strategies during sidestepping. Scand J Med Sci Sport. 2017;27(5):492-500. doi:10.1111/sms.12668

60. Lee MJC, Lloyd DG, Lay BS, Bourke PD, Alderson JA. Effects of different visual stimuli on postures and knee moments during sidestepping. Med Sci Sports Exerc. 2013;45(9):17401748. doi:10.1249/MSS.0b013e318290c28a 


\section{Tables}

Table 1. Significant differences between PRE- and POST-intervention biomechanical variables. Means reported for the phase in which a significant difference between groups was identified.

\begin{tabular}{|c|c|c|c|c|c|c|c|c|}
\hline \multicolumn{3}{|c|}{ Variable } & \multirow{2}{*}{$\begin{array}{c}\text { Phase } \\
\text { (\%) } \\
58-100\end{array}$} & \multirow{2}{*}{$\begin{array}{c}\begin{array}{c}\text { Mean } \pm \text { SD } \\
\text { PRE }\end{array} \\
203 \pm 81.5\end{array}$} & \multirow{2}{*}{$\begin{array}{c}\text { Mean } \pm \text { SD } \\
\text { POST } \\
266 \pm 75.4\end{array}$} & \multirow{2}{*}{$\begin{array}{c}\text { Mean \% } \\
\text { change } \\
\text { PRE to } \\
\text { POST } \\
n / a\end{array}$} & \multirow{2}{*}{$\begin{array}{c}\mathbf{p} \\
<0.001\end{array}$} & \multirow{2}{*}{$\begin{array}{r}\begin{array}{c}\text { Effect } \\
\text { size }\end{array} \\
0.58\end{array}$} \\
\hline COM & $\begin{array}{l}\text { Distance to COP } \\
(\mathrm{mm})\end{array}$ & $A-P$ & & & & & & \\
\hline Pelvis & Angle $\left({ }^{\circ}\right)$ & Transverse & $0-100$ & $35.2 \pm 11.4$ & $45.4 \pm 11.9$ & $\mathrm{n} / \mathrm{a}$ & $<0.001$ & 0.66 \\
\hline \multirow{3}{*}{ Ankle } & Angle $\left({ }^{\circ}\right)$ & Sagittal & $27-89$ & $19.4 \pm 9.2$ & $24.6 \pm 8.4$ & $\mathrm{n} / \mathrm{a}$ & $<0.001$ & 0.67 \\
\hline & Moment (N.cm. $\left.\mathrm{kg}^{-1}\right)$ & Sagittal & $64-80$ & $29.4 \pm 6.7$ & $33.8 \pm 6.2$ & 15.1 & 0.003 & 0.67 \\
\hline & Power (W.kg $\left.{ }^{-1}\right)$ & Sagittal & $79-88$ & $9.4 \pm 3.7$ & $11.8 \pm 3.8$ & 25.8 & $<0.001$ & 0.66 \\
\hline
\end{tabular}

Note. $\mathrm{PRE}=$ pre-intervention; POST $=$ post-intervention; $\mathrm{COM}=$ centre of body mass; COP = centre of pressure; $\mathrm{A}-\mathrm{P}=$ antero-posterior; $\mathrm{SD}=$ standard deviation. Effect sizes reported are Cohen's $\mathrm{d}$ standardised effect size. Percentage changes are reported only for those variables that should be interpreted as ratio data in this context.

Table 2. PRE- and POST-intervention performance variables

\begin{tabular}{|c|c|c|c|c|c|}
\hline Variable & PRE mean \pm SD & POST mean \pm SD & $\begin{array}{c}\text { Mean \% } \\
\text { change PRE to } \\
\text { POST }\end{array}$ & $\mathbf{p}$ & $\begin{array}{l}\text { Effect } \\
\text { size }\end{array}$ \\
\hline Total time (s) & $1.86 \pm 0.12$ & $1.83 \pm 0.10$ & -2.1 & $0.06 \mathrm{NS}$ & 0.27 \\
\hline Contact time (s) & $0.34 \pm 0.05$ & $0.34 \pm 0.05$ & 0 & $0.97 \mathrm{NS}$ & 0.00 \\
\hline $\begin{array}{l}V_{\text {COMH }} \text { at start of } \\
\text { stance }\left(\mathrm{ms}^{-1}\right)\end{array}$ & $2.82 \pm 0.30$ & $2.86 \pm 0.28$ & 0.04 & $0.55 \mathrm{NS}$ & 0.14 \\
\hline $\begin{array}{l}V_{\text {comH }} \text { at end of } \\
\text { stance }\left(\mathrm{ms}^{-1}\right)\end{array}$ & $2.64 \pm 0.22$ & $2.69 \pm 0.21$ & 0.05 & $0.23 \mathrm{NS}$ & 0.23 \\
\hline
\end{tabular}

Note. $\mathrm{PRE}=$ pre-intervention; $\mathrm{POST}=$ post-intervention; $\mathrm{V}_{\mathrm{COMH}}=$ horizontal velocity of the body centre of mass. Effect sizes reported are Cohen's d standardised effect size. 
Table 3. Hip And Groin Outcome Score (HAGOS) results

\begin{tabular}{lcccc}
\hline \multicolumn{1}{c}{ Subscale } & PRE & POST & p & Effect size \\
\hline Symptoms & $63.2 \pm 15.3$ & $78.9 \pm 11.3$ & $<0.001$ & 1.17 \\
Pain & $76.5 \pm 13.4$ & $87.2 \pm 9.9$ & $<0.001$ & 0.91 \\
ADL & $77.2 \pm 16.5$ & $90.1 \pm 11.1$ & $<0.001$ & 0.92 \\
Sport and Rec & $52.3 \pm 15.8$ & $80.3 \pm 13.7$ & $<0.001$ & 1.89 \\
PA & $21.4 \pm 21.7$ & $50.7 \pm 28.5$ & $<0.001$ & 1.16 \\
QOL & $36.1 \pm 16.6$ & $55.0 \pm 18.9$ & $<0.001$ & 1.07
\end{tabular}

Note. $\mathrm{PRE}=$ pre-intervention; $\mathrm{POST}=$ post-intervention; $\mathrm{ADL}=$ activities of daily living; Sport and Rec $=$ sports and recreational activities; $\mathrm{PA}=$ participation in physical activity; $\mathrm{QOL}=$ quality of living. Effect sizes reported are Cohen's d standardised effect size. PRE $=$ pre-intervention; POST $=$ postintervention 


\section{Figures}

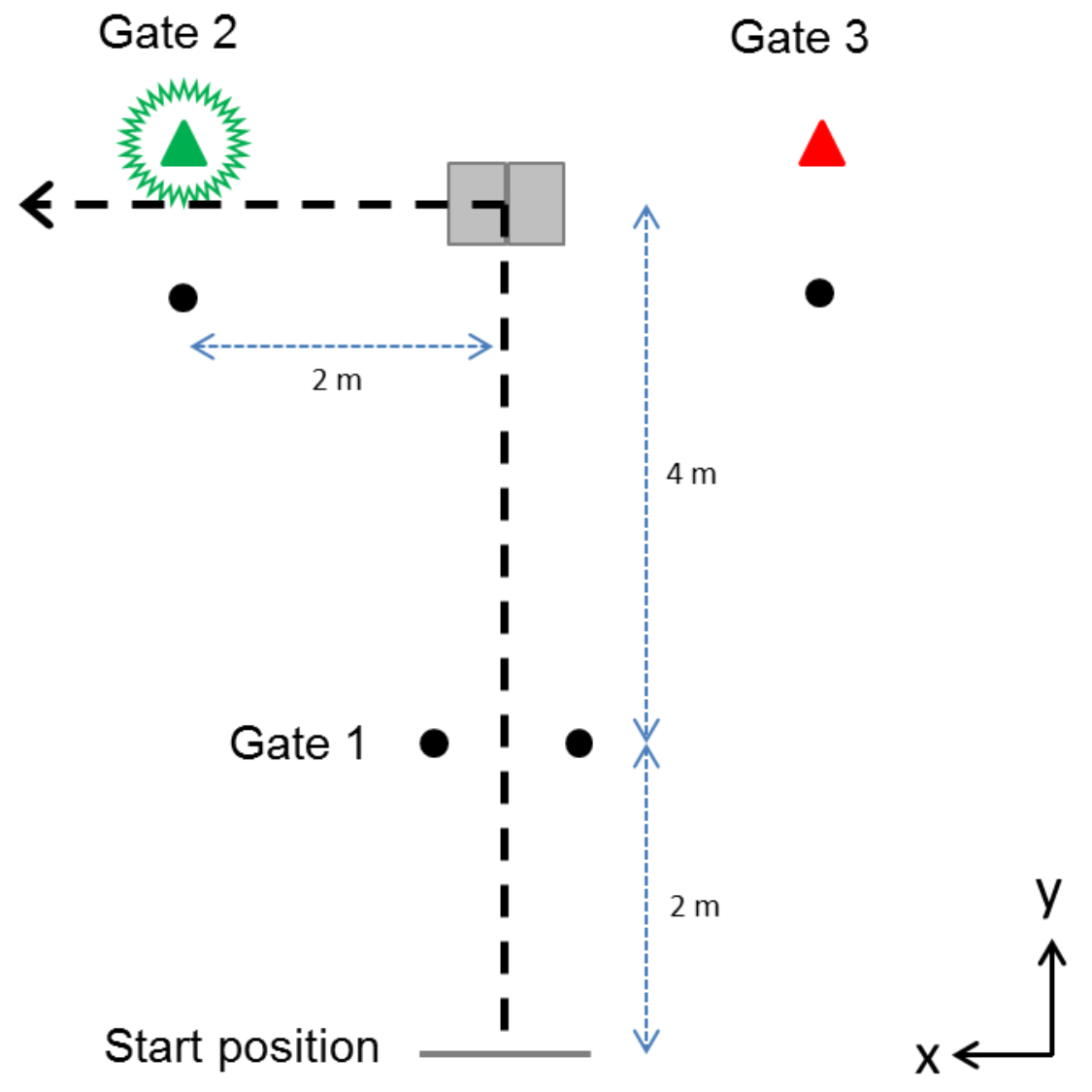

Figure 1. The experimental task (not to scale). Three timing gates were used, the first to trigger the direction signal and start the time recording ('Gate 1'; black filled circles) and the others ('Gate 2' and 'Gate 3') to signal turns to the left and the right respectively (coloured triangles indicate the location of the signal light; black filled circles indicate the corresponding boundary of each gate). The force platforms are marked schematically as grey filled rectangles. The laboratory global $\mathrm{x}$ and $\mathrm{y}$ axes are marked and defined in the lower right of the figure; $\mathrm{z}$ is vertical. The thick broken line indicates an example trial in which the participant cut from the right leg, responding to a visual signal at Gate 2. 

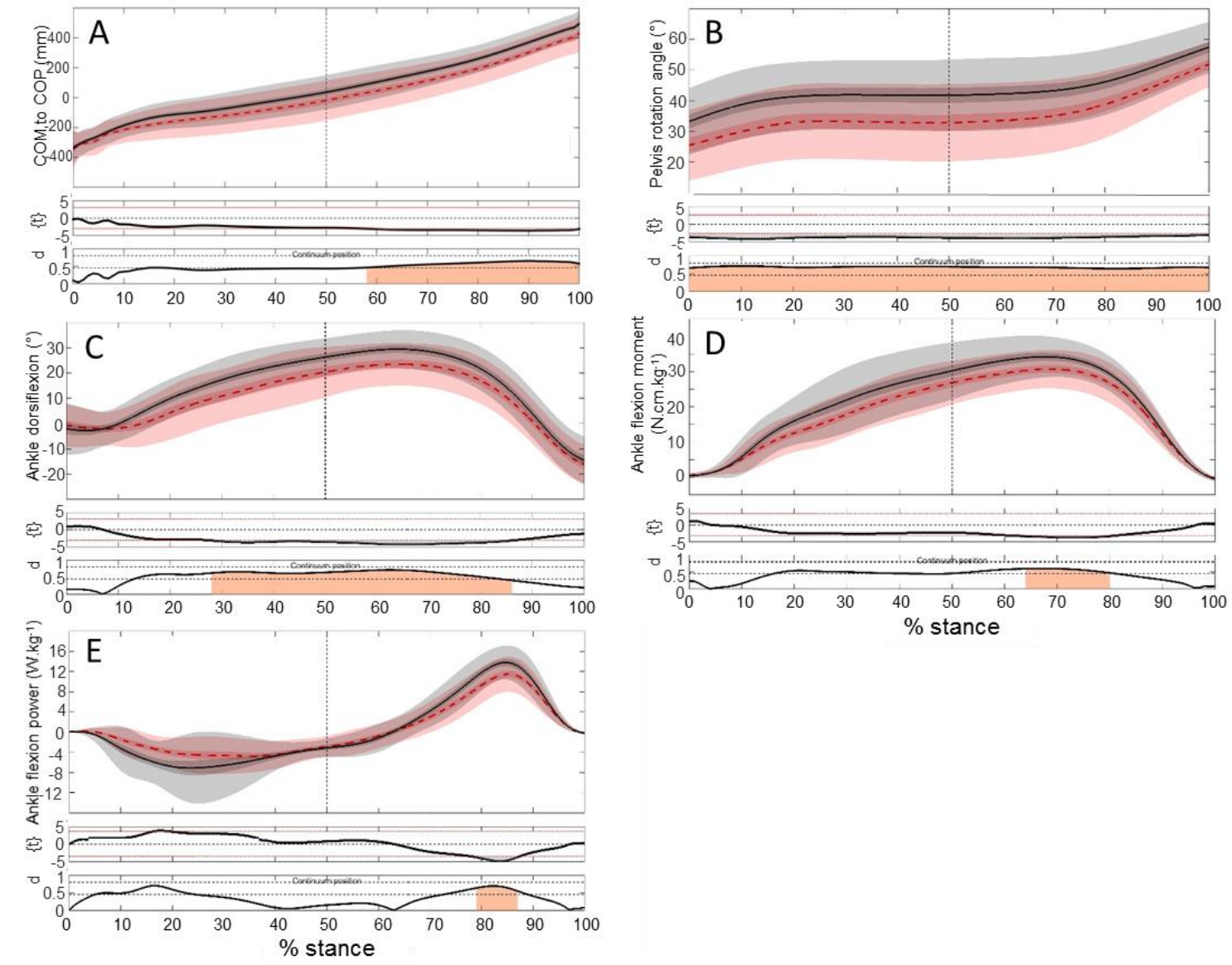

Figure 2. Graphical representations of biomechanical variables with significant PRE-POST changes. (A) horizontal distance from the COM to the COP in the sagittal plane; (B) pelvis angle in the transverse plane; (C) ankle angle in the sagittal plane; (D) ankle moment in the sagittal plane; (F) ankle power in the sagittal plane. Dashed red line represents PREintervention mean; unbroken black line represents POST-intervention mean. Shading represents $\pm 1 \mathrm{SD}$ for each condition. The lower panels in each subfigure report the SPM $\{\mathrm{t}\}$ curves (middle panel) and pointwise Cohen's d standardised effect size (bottom panel), with regions of significant difference (i.e. where $\operatorname{SPM}\{t\}$ exceeds the critical threshold) indicated with orange shading. 